\title{
Commentary: Cell-based therapy for chronic lung allograft dysfunction: Patient selection remains the conundrum
}

\author{
Mara B. Antonoff, MD
}

\author{
From the University of Texas MD Anderson Cancer Center, Houston, Tex. \\ Disclosures: Author has nothing to disclose with regard to commercial support. \\ Received for publication Jan 15, 2019; accepted for publication Jan 15, 2019; available ahead of print Feb 20, \\ 2019. \\ Address for reprints: Mara B. Antonoff, MD, 1400 Pressler St, Unit 1489, Houston, TX 77030 (E-mail: \\ mbantonoff@mdanderson.org). \\ J Thorac Cardiovasc Surg 2019;157:2540-1 \\ $0022-5223 / \$ 36.00$ \\ Copyright (c) 2019 by The American Association for Thoracic Surgery \\ https://doi.org/10.1016/j.jtcvs.2019.01.058
}

For patients undergoing lung transplantation, avoidance and management of chronic lung allograft dysfunction (CLAD) are of extreme importance in determining long-term quality of life and survival. ${ }^{1}$ Identifying patients at risk for this disease process and implementing strategies to treat them proactively could ultimately alter the survival outcomes for large numbers of transplant recipients. Transplant arteriosclerosis (TA), which is mediated by alloimmune targeting of the graft, is closely tied to CLAD and is accompanied by intimal hyperplasia and vasomotor dysfunction. ${ }^{2}$ Models of TA thus potentially serve to aid us in better understanding the pathology of CLAD, as a foundation for important translational studies.

In 2016, Siemeni and colleagues ${ }^{3}$ reported on their in vivo development of TA in a humanized mouse model. By transplanting segments of human pericardiophrenic arteries from lung donors as aortic interposition grafts into immune-deficient mice, these investigators were able to prompt TA in the mice. Moreover, they showed that alloantigen priming with the injection of peripheral blood mononuclear cells from the recipient led to more severe TA, but that this process could be suppressed by the provision of peripheral blood regulatory $\mathrm{T}$ cells. This model has a number of strengths, theoretically showing functional evidence for the allograft-protective potency of human endogenous regulatory $\mathrm{T}$ cells in lung transplant recipients. Translating these findings to human patients, however, remained an important void.

In the latest study from Siemeni and colleagues, ${ }^{2}$ published in this issue of the Journal, efforts have been made to correlate development of TA in mice by using their previously described model with grafts from human lung recipients with subsequent CLAD development. Siemeni and colleagues $^{2}$ propose, and then successfully demonstrate, that TA at 28 days was significantly more severe among mice for whom the respective patients had CLAD develop during approximately the next 2 years. In other words, it was possible to use the findings from the mice after only 4 weeks to predict which patients would be at greater risk

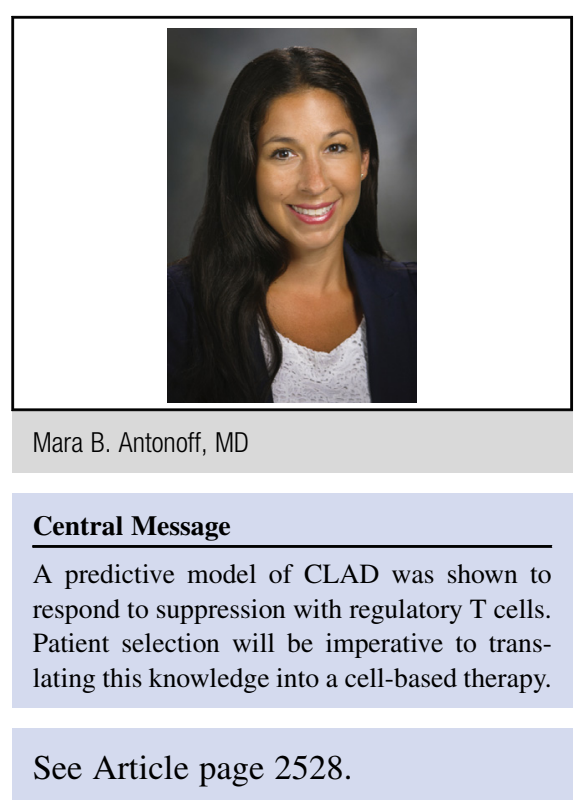

for CLAD development many months down the line. Furthermore, through a series of elegant experiments, Siemeni and colleagues ${ }^{2}$ were able to show that enrichment with regulatory $\mathrm{T}$ cells could suppress the development of TA both in mice with grafts from human patients in whom CLAD developed and in mice with grafts from patients without CLAD development.

Siemeni and colleagues ${ }^{2}$ are to be commended for their continued quest to further elucidate the early events in CLAD development. In this article, they conclude that TA remains sensitive to inhibition by autologous regulatory $\mathrm{T}$ cells, and that a cell therapy-based approach for the prevention and treatment of CLAD after lung transplantation may be on the horizon. This represents an exciting notion, and studies such as that of Siemeni and colleagues ${ }^{2}$ clearly help make progress in this direction, but we still have an enormous hurdle to address: To which patients would we provide such a tailored, resource-intense treatment? Clearly, one needs to be able to predict CLAD clinically to use such a cell-based therapy. Yes, Siemeni and colleagues $^{2}$ have shown that the development of TA in mice can predict the development of CLAD in human patients, but is this realistically a feasible means of predicting CLAD in the clinical setting? Unfortunately, this remains a challenge. To provide a cell-based therapy that takes advantage of the suppressive activities of regulatory T cells, 
one would need either to be prepared to deliver such a treatment to all recipients empirically or to determine which patients are at greatest risk of CLAD on the basis of clinically available characteristics (not the development of TA in a corresponding mouse).

Thus, although great progress has been made, we still struggle with an ever-present conundrum in our field. As we innovate to create novel therapies that will be efficacious in some patients, how do we predict who will need the therapy and who will benefit the most? This is a familiar problem for thoracic surgeons. Which patients will respond to immunotherapy before lung resection, and who will have progression? Which of our patients will benefit from adjuvant chemotherapy after esophagectomy, and who will not? It is clear that some patients will fare well regardless of any additional treatments that we can offer them, and, likewise, there are also groups of individuals who will, unfortunately, fare poorly despite any maximal interventions that we may provide. The challenge remains to target those patients for whom there is a potential to modify outcomes with our interventions. Although Siemeni and colleagues ${ }^{2}$ have continued to produce outstanding work, leading to a greater understanding of the immunologic environment in CLAD, it is clear that translation into human therapy still requires addressing many more unanswered questions regarding optimal candidates for treatment. Nonetheless, as has been said by many a wise scholar, an outstanding article raises more questions than it answers.

\section{References}

1. Verleden SE, Todd JL, Sato M, Palmer SM, Martinu T, Pavlisko EN, et al. Impact of CLAD phenotype on survival after lung retransplantation: a multicenter study. Am J Transplant. 2015;15:2223-30.

2. Siemeni T, Knöfel AK, Ius F, Sommer W, Salman J, Böthig D, et al. Transplant arteriosclerosis in humanized mice reflects chronic lung allograft dysfunction and is controlled by regulatory T cells. J Thorac Cardiovasc Surg. 2019;157: 2528-37.

3. Siemeni T, Knöfel AK, Madrahimov N, Sommer W, Avsar M, Salman J, et al. In vivo development of transplant arteriosclerosis in humanized mice reflects alloantigen recognition and peripheral Treg phenotype of lung transplant recipients. Am J Transplant. 2016;16:3150-62. 